\title{
An enhanced electrical angle representation in PMSM control with misplaced Hall-effect switch sensors
}

\author{
Carlos Miguel-Espinar ${ }^{1}$, Daniel Heredero-Peris ${ }^{1}$, Gabriel Igor-Gross ${ }^{1}$, Marc Llonch-Masachs ${ }^{1}$, and \\ Daniel Montesinos-Miracle ${ }^{1}$ \\ ${ }^{1}$ Centre d'Innovació Tecnològica en Convertidors Estàtics i Accionaments (CITCEA-UPC) ETS d'Enginyeria Industrial de \\ Barcelona, Av. Diagonal 647, 08028 Barcelona, Spain, Phone: +34 934016727
}

\begin{abstract}
This paper proposes an enhanced method to represent the rotor position to control a Permanent Magnet Synchronous Motor (PMSM) based on a First-Order TaylorSeries approximation, by using misplaced Hall-effect switch sensors. The main feature of this algorithm is the speed estimation from two consecutive edge-transitions of a single phase. Furthermore, when a new electrical cycle starts, a compensation algorithm assists this method to achieve error cancellation in the electrical angle representation. Simulation results with an Interior Permanent Magnet Synchronous Motor (IPMSM) verify the effectiveness of the proposed method showing less than $0.5 \%$ error in the angle estimation and less than $2 \%$ in terms of harmonic content.
\end{abstract}

Index Terms - First-Order Taylor-Series, Hall-effect switch sensor, Hall-effect switch sensor offset, Permanent magnet motors.

\section{INTRODUCTION}

During the last years, the use of micro-mobility in congested urban areas has undergone enormous growth. Escooters and e-motorbikes has a Permanent Magnet Synchronous Motor (PMSM) as the central traction component, which is preferable because of its high-power density, easy maintenance, reduced size, and high efficiency [1]. Its control algorithm is based on Field Oriented Control algorithms, which makes mandatory the knowledge of the rotor position.

Hall-effect switch sensors are worthwhile in micromobility applications, more than encoders or resolvers, to achieve low-cost and compact systems. In recent years, some authors have developed algorithms to detect the rotor position with sensorless methods [2]. They are classified into two categories: Back Electromotive Force Methods (BEFMs) and High-Frequency Current Injection Methods (HFCIMs). BEFMs show drawbacks at very low and zero speeds. Meanwhile, HFCIMs require the usage of a considerable portion of the DC bus voltage, making them susceptible to different load conditions. Other authors have studied the possibility of mixing sensorless methods with Hall-effect sensors to generate the electrical angle; therefore, to improve the performance of preceding techniques [3]-[6]. Nevertheless, the need for complex mathematical algorithms and its corresponding computational burdens, and the high dependence on motor parameters make them difficult for the real implementation.
This paper is focused on the use of Hall-effect switch sensors which present two main challenges: low speed and misplaced sensors. The low speed issue is solved by applying the $120^{\circ} \mathrm{E}$ Square-Wave Commutation Method. It is applied until the spinning of the magnets is high enough to represent the electrical angle as a continuous waveform [3]. Different techniques are developed to solve the misplaced problem. In [7], a linear interpolation, based on the least-squares method, is used to represent the electrical angle. It only considers the motor performance at constant speed and the gradual method adjustment introduces some deviations in the electrical angle and the electrical speed. Besides, this method requires a huge microcontroller burden. In [8], a moving average with the same Hall-sensor edge is considered, but the estimated speed is calculated once an electrical turn, and it does not correct the phase-shift between Hall-sensors and windings.

Along with the challenges above, the algorithm to correct the electrical angle, when a new Hall-effect sensor edge is achieved, is essential. Some authors propose immediately correct the angle, provoking an instantaneous change in the current waveform and therefore, the torque applied to the motor. Others correct the angle averagely in the next electrical sector, by supposing that the time in the next sector will be the same as the previous sector, [7], [8]. This method provokes a harmonic content in the current waveform and a variation in the estimated speed.

This paper proposes an algorithm to represent the electrical angle by considering the misplacement of Halleffect switch-sensors. A no-load test as a generator is required to measure only once the phase-shift angle between sensors and windings. Then, the assigned electrical angle to each edge is corrected with this phaseshift angle. So, the continuous electrical angle representation is estimated by a First-Order Taylor-Series approximation, from which the speed is calculated based on the time elapsed between two consecutive edges from the same phase. Besides, when a new electrical period is reached, the phase-shift angle is corrected by means of a speed change in a determined number of electrical sectors.

The content of this paper is organized into six sections. Section II explains the effect of misplaced Hall-effect switch sensors. Section III presents the proposed speed estimation method. Section IV describes the electrical angle representation, as well as the compensation 
algorithm. In Section V, some simulation results claim the effectiveness of this method. Finally, Section VI describes the conclusion achieved after this research was conducted.

\section{MISPLACED HALL-EFFECT SWITCH SENSORS}

\section{A. Ideal Hall signals without offsets}

The electrical rotor position is determined each $60^{\circ} \mathrm{E}$ due to three Hall-effect switch sensors, as long as these are precisely separated $120^{\circ} \mathrm{E}$ from each other. This information is repeated pair of poles times along a mechanical turn provided that the permanent magnets are well-distributed around the rotor surface. As the technology to assemble the stator lamination sheets with the Hall sensors is not simple, it cannot guarantee the precise position of them.
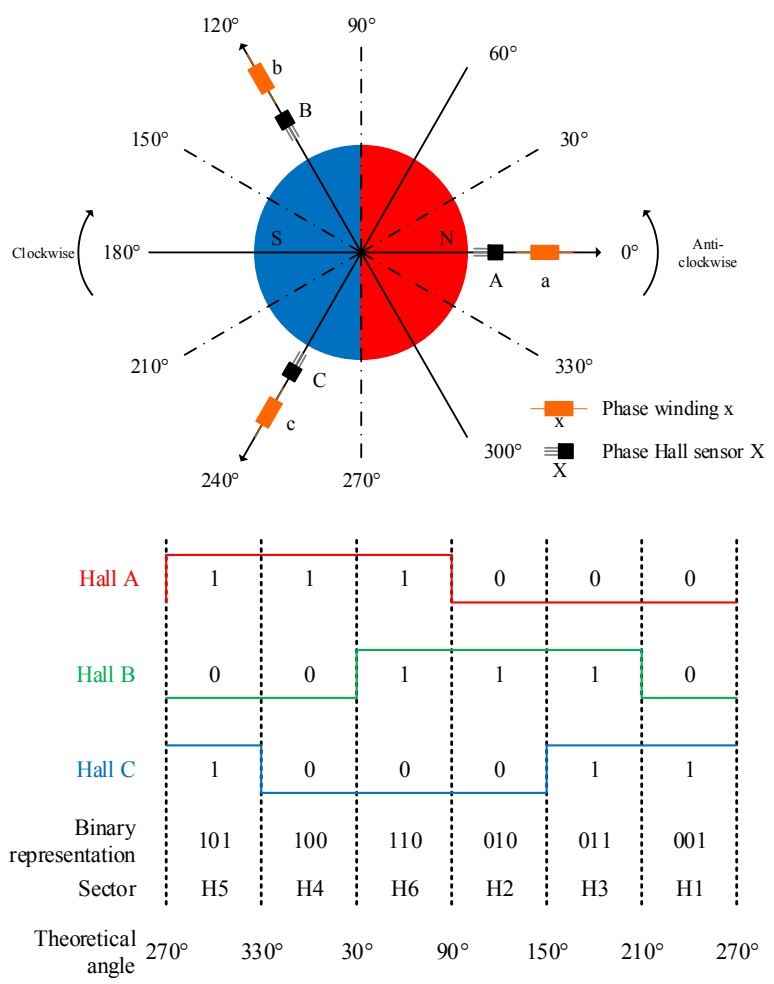

Fig. 1a). Polar diagram of a single electrical period. No phase-shift between Hall sensors and stator windings. b). Time diagram of Hall sensor signals in anti-clockwise direction (red, green, and blue lines for phases $\mathrm{A}, \mathrm{B}$, and $\mathrm{C}$, respectively), as well as, a binary representation of the electrical sector, and the theoretical electrical angle at each edge.

Fig. 1a) shows the polar diagram of one electrical turn, which is represented by a pair of poles. The winding and the Hall sensor for each phase are well-located, and no phase-shift is considered. The rotor, which is represented by two magnets (north and south), can rotate in the clockwise direction or the anti-clockwise direction.

Fig. 1b) shows the timing diagram of the Hall sensor signals (red, green, and blue lines for phases A, B, and C, respectively) when the rotor spins in the anti-clockwise direction. The output signal of a switch sensor is electrically defined as high-level or low-level, representing a binary signal. From a digital system, when the output signal is at a high-level, the value is 1 , and when it is at a low-level, the value is 0 .

In Fig. 1b) the edge between the high and low level, and vice versa, for each output depends on the magnet position. The three signals are gathered to form a binary representation of the electrical sector. The sector number is calculated by considering the most and the least significant bit from the phase A and phase C, respectively. If no offset is considered between Hall sensors and windings, then two consecutive edges are separated $60^{\circ} \mathrm{E}$.

If the motor is spinning and the digital controller detects a new sector, the assigned electrical angle only depends on the type of angle representation. At low speed, the electrical angle is represented in a discrete way following a $120^{\circ} \mathrm{E}$ commutation technique, which is used to control a Brushless DC Motor. Nevertheless, at high speed, this angle is represented in a continuous way to avoid current ripple, and so to diminish the noise and vibrations.

TABLE I shows the assigned electrical angle $\left(\theta_{\mathrm{e}(\mathrm{Hall}(\mathrm{z}))}\right)$ when a new sector is detected. In the discrete representation, the electrical angle coincides with the midangle of each sector. This strategy gets the minimum angle-error and torque-ripple over a specific sector. However, in the continuous representation the updated angle depends on the turning direction.

TABLE I

\begin{tabular}{|c|c|c|c|}
\hline \multicolumn{4}{|c|}{ ELECTRICAL ANGLE ASSIGNMENT } \\
\hline \multirow[b]{2}{*}{$\begin{array}{c}\text { Hall Sector } \\
\text { Hall(z) }\end{array}$} & \multirow[b]{2}{*}{$\begin{array}{c}\text { Discrete } \\
\theta_{\mathrm{e}(\text { Hall(z)) }}\left[{ }^{\circ} \mathbf{E}\right]\end{array}$} & \multicolumn{2}{|c|}{ Continuous } \\
\hline & & $\begin{array}{c}\text { Clockwise } \\
\theta_{\mathrm{e}(\operatorname{Hall}(\mathrm{z}))}\left[{ }^{0} \mathbf{E}\right]\end{array}$ & $\begin{array}{c}\text { Anti-clockwise } \\
\theta_{\mathrm{e}(\mathrm{Hall}(\mathrm{z}))}\left[{ }^{\circ} \mathrm{E}\right]\end{array}$ \\
\hline 1 & 240 & 270 & 210 \\
\hline 2 & 120 & 150 & 90 \\
\hline 3 & 180 & 210 & 150 \\
\hline 4 & 0 & 30 & 330 \\
\hline 5 & 300 & 330 & 270 \\
\hline 6 & 60 & 90 & 30 \\
\hline
\end{tabular}

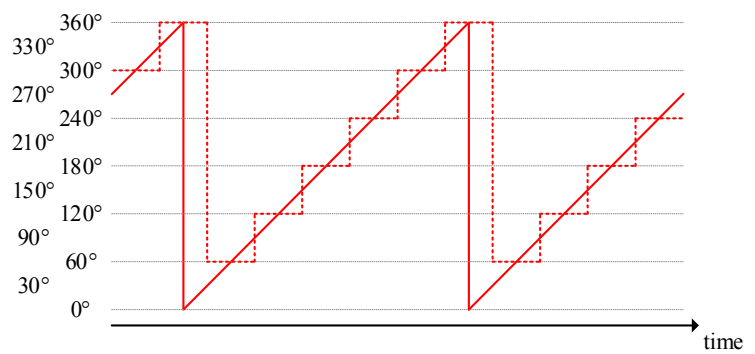

Fig. 2. Electrical angle representation without angle offset (red dashed line is the discrete representation, and red solid line is the continuous representation).

Fig. 2 shows the electrical angle representation regarding the time. In dashed and solid line are represented the discrete and continuous electrical angle, respectively. This representation is the main objective of the proposed method because this lets the inverter no generate current harmonic content due to the electrical angle representation. The transition between the discrete and continuous 
representation is defined to avoid any instantaneous change in the electrical angle.

\section{B. Hall signal offset drawbacks}

Due to the mechanical errors either in the manufacturing or assembling process, each Hall sensor may be misplaced a certain mechanical angle regarding its corresponding phase winding. These errors will negatively affect the electrical angle representation. And as a consequence, the performance of the torque and speed controllers will be affected, deriving in vibrations, noise, and unstable controllability.

Fig. 3 represents the polar diagram of misplaced Hall sensor signals in one electrical period. The red, green, and blue lines symbolize the Hall sensor signals for phases A, $\mathrm{B}$, and $\mathrm{C}$, respectively. In this example, all Hall sensors are misplaced to its corresponding winding phase. On the one hand, the black lines define the electrical base if the motor windings are considered. On the other hand, the brown lines shape the electrical base if the Hall sensor signals are regarded. As can be seen, the Hall sensor base is not wellbalanced and misplaced.

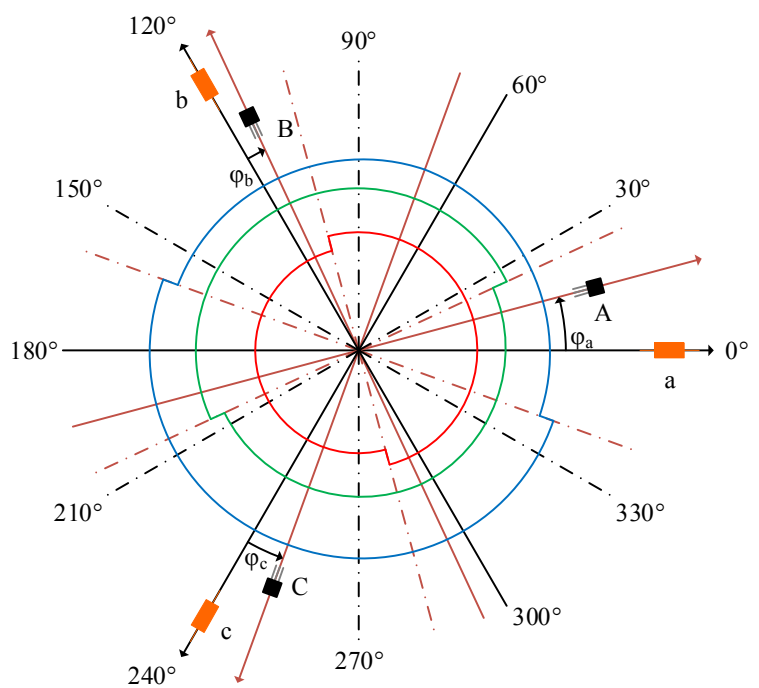

Fig. 3. Circle diagram of misplaced Hall sensors signals in one electrical period. The solid and dot-dash black lines refer to the stator windings base. The solid and dot-dash brown lines indicate the rotor base created from the Hall sensor signals. Red, green, and blue lines refer to the Hall signals of phases A, B, and C, respectively.

It has been considered an electrical angle $\varphi_{\mathrm{a}}, \varphi_{\mathrm{b}}$, and $\varphi_{\mathrm{c}}$ as the angle offset between the phase winding and its analogous Hall sensor for phases A, B, and C, respectively. The specified electrical angles in Fig. 3 corresponds to those if the motor windings were the electrical base, from the power converter point of view.

So, the assigned value for each Hall sensor edge will be different from the theoretical angle expressed in TABLE I, and an angle offset is added according to TABLE II. In doing so, the electrical angle information becomes its real value. The angle offset in each sector is different if the spinning direction is clockwise or anti-clockwise because the edge transition comes from a distinct phase.

Fig. 4 shows the rising and falling edges both in the case of well-located and misplaced Hall sensors, in red, green, and blue color for phases A, B, and C, respectively. In red line is represented the ideal continuous wave of the electrical angle if no offset is considered. However, an offset angle should be applied when an edge is gotten, and misplaced sensors are considered. The angle offsets $\varphi_{\mathrm{a}}, \varphi_{\mathrm{b}}$, and $\varphi_{\mathrm{c}}$ are the same both in north and south pole, and in different electrical periods, as long as the magnets are the same, and they are well-distributed along the rotor surface. $\theta_{\mathrm{e}}$ represents the electrical angle of the rotor, $\mathrm{e}_{(\mathrm{i})}$ (for $\forall \mathrm{i} \geq$ 1) symbolizes each one of the Hall sensor edges, $\Delta t_{(i)}$ and $\Delta t^{\prime}{ }_{(i)}$ is the elapsed time between two consecutive Hall sensor edges either offset angle is considered or not, respectively, and $\Delta \mathrm{t}_{\mathrm{Hall}(\mathrm{x}, \mathrm{j})}$ and $\Delta \mathrm{t}^{\prime}{ }_{\mathrm{Hall}(\mathrm{x}, \mathrm{j})}$ (for $\forall \mathrm{x}=\mathrm{A}, \mathrm{B}$ or $\mathrm{C}$, and $\forall \mathrm{j} \geq 1$ ) is the elapsed time between two successive Hall sensor edges of the same phase either offset angle is considered or not, respectively.

TABLE II

SHIFTED ELECTRICAL ANGLE ASSIGNMENT

\begin{tabular}{|l|r|r|}
\hline \multirow{2}{*}{$\begin{array}{c}\text { Hall Sector } \\
\text { Hall(z) }\end{array}$} & \multicolumn{2}{|c|}{ Shifted continuous angle } \\
\cline { 2 - 3 } & $\begin{array}{c}\text { Clockwise } \\
\boldsymbol{\theta}_{\mathbf{e}(\mathbf{H a l l}(\mathbf{z})}\left[{ }^{\mathbf{0}} \mathbf{E}\right]\end{array}$ & $\begin{array}{c}\text { Anti-clockwise } \\
\boldsymbol{\theta}_{\mathbf{e}(\mathbf{H a l l}(\mathbf{z}))}\left[{ }^{\mathbf{0}} \mathbf{E}\right]\end{array}$ \\
\hline 1 & $270+\varphi_{\mathrm{A}}$ & $210+\varphi_{\mathrm{B}}$ \\
\hline 2 & $150+\varphi_{\mathrm{C}}$ & $90+\varphi_{\mathrm{A}}$ \\
\hline 3 & $210+\varphi_{\mathrm{B}}$ & $150+\varphi_{\mathrm{C}}$ \\
\hline 4 & $30+\varphi_{\mathrm{B}}$ & $330+\varphi_{\mathrm{C}}$ \\
\hline 5 & $330+\varphi_{\mathrm{C}}$ & $270+\varphi_{\mathrm{A}}$ \\
\hline 6 & $90+\varphi_{\mathrm{A}}$ & $30+\varphi_{\mathrm{B}}$ \\
\hline
\end{tabular}

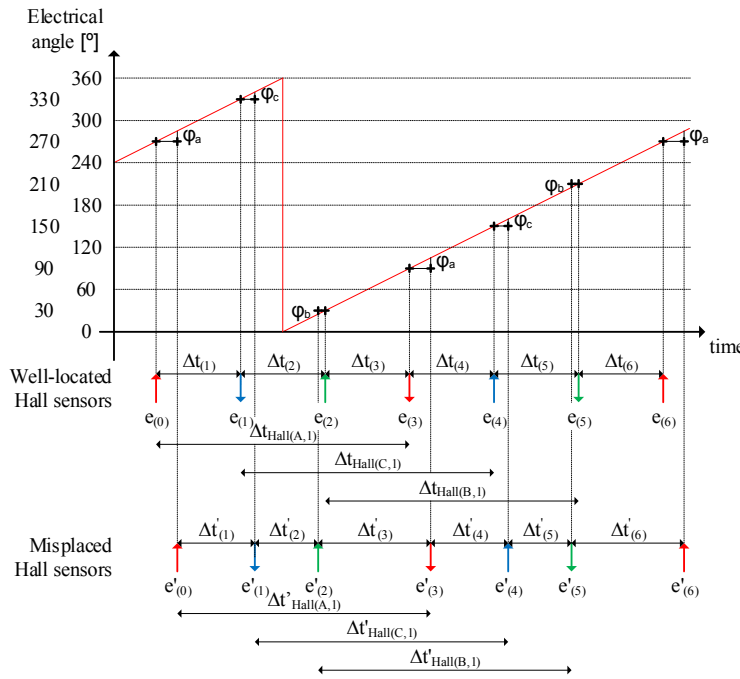

Fig. 4. Hall sensor edges with well-located and misplaced sensors. The red line is the ideal electrical angle representation, and the red, green, and blue rising and falling edges correspond to Hall sensor phase $\mathrm{A}, \mathrm{B}$ and $\mathrm{C}$, respectively.

\section{SPEED ESTIMATION FROM HALL SIGNALS}

If the Hall sensors are well-located and the motor rotates at a constant speed, the equation to calculate the velocity from the difference between two consecutive edges is 


$$
\omega_{e(i)}=\frac{\frac{\pi}{3}}{\Delta t_{(i-1)}} \forall i \geq 2,
$$

where $\omega_{\mathrm{e}(\mathrm{i})}$ is the electrical speed estimated for the period $\mathrm{i}$, and $\Delta \mathrm{t}_{(\mathrm{i}-1)}$ is the elapsed time between two consecutive edges during the period $\mathrm{i}-1$.

The main problem with misaligned sensors is the error calculation of the estimated speed employing the Eq. (1). The real-time between two consecutive edges may be higher or smaller than the theoretical value at a constant speed, as can be seen in Fig. 4. Therefore, $\omega_{\mathrm{e}}$ '(i) is different from $\omega_{\mathrm{e}(\mathrm{i})}$, where $\omega_{\mathrm{e}}{ }^{\prime}(\mathrm{i})$ is the electrical speed calculated from $\Delta \mathrm{t}^{\prime}{ }_{(\mathrm{i}-1)}$ using the Eq. (1).

Notwithstanding, it can be demonstrated that the elapsed time between two consecutive edges from the same sensor is not affected by the misplaced position, as long as the permanent magnets have the same size, and they are welldistributed along the rotor surface. This is an indication that the electrical periods are invariable in an entire mechanical turn. So, the time between two consecutive edges of the same phase, if no offset is considered, is expressed as

$$
\begin{aligned}
& \Delta t_{H \operatorname{all}(x, j)}=\Delta t_{(i)}+\Delta t_{(i+1)}+\Delta t_{(i+2)}, \\
& \forall x=A, B, \text { or } C, \quad \forall j \geq 1, \text { and } \forall i \geq 1 ;
\end{aligned}
$$

where the subscript $\mathrm{x}$ refers to the phase sensor that can be $\mathrm{A}, \mathrm{B}$, or $\mathrm{C}$, and $\mathrm{j}$ indicates the number of periods of the same phase and $i=3 j-2$.

Meanwhile, the time between two consecutive edges from the same phase with misplaced Hall sensors is calculated as

$$
\begin{aligned}
& \Delta t^{\prime}{ }_{\text {Hall }(x, j)}=\Delta t^{\prime}{ }_{(i)}+\Delta t^{\prime}{ }_{(i+1)}+\Delta t^{\prime}{ }_{(i+2)}, \\
& \forall x=A, B \text {, or } C, \quad \forall j \geq 1, \text { and } \forall i \geq 1 .
\end{aligned}
$$

Therefore, $\Delta t_{\text {Hall }(x, j)}$ is equal to $\Delta t_{\text {Hall }(x, j)}^{\prime}$ independently of $\varphi_{\mathrm{a}}, \varphi_{\mathrm{b}}$ and $\varphi_{\mathrm{c}}$. In this manner, the estimated speed can be calculated from the elapsed time in half of each electrical period as

$$
\omega_{e_{(i)}}=\frac{\pi}{\Delta t_{(i-1)}+\Delta t_{(i-2)}+\Delta t_{(i-3)}}, \quad \forall i \geq 4 .
$$

This algorithm is named as the moving average with three samples, and it lets the electrical angle representation not to be affected by the offset angle. Furthermore, the response time is half compared with the proposed algorithm in [8].

\section{POSITION ESTIMATION AND COMPENSATION ALGORITHM}

During the performance at $120^{\circ} \mathrm{E}$ commutation algorithm, the assigned electrical angle is the same as TABLE I indicates for the discrete case, depending on the electrical sector in which the rotor is located.

At the moment when the number of pulses is higher than six (an entire electrical turn), the angle is continuously calculated according to a Uniform Angular Movement. The average speed of one electrical sector is considered constant, and this is calculated from the previous midelectrical period following the Eq. (4). So, the discrete angle representation is computed as

$$
\begin{aligned}
\theta_{e(k)}=\theta_{e(k-1)}+\omega_{e(i)} & \cdot\left(t_{(k)}-t_{(k-1)}\right) \\
& \forall k \geq 1, \quad \forall i \geq 4 ;
\end{aligned}
$$

where, $\theta_{\mathrm{e}(\mathrm{k})}$ and $\theta_{\mathrm{e}(\mathrm{k}-1)}$ are the electrical angles at the discrete instants $\mathrm{k}$ and $\mathrm{k}-1$, respectively; and $\mathrm{t}_{(\mathrm{k})}-\mathrm{t}_{(\mathrm{k}-1)}$ is defined as the sample period at which the angle is calculated.

The electrical speed is calculated whenever a new Hall sensor edge is detected and as of two consecutive edges have occurred. Nonetheless, the electrical angle is calculated at a high-frequency time basis. Generally, the control loop period is chosen as a sample period to update this angle.

The estimated position according to the Eq. (5) can be different from the theoretical value defined in TABLE II, mainly during the acceleration and deacceleration transients. Some authors have considered updating the misleading angle immediately at each Hall sensor transition. Nevertheless, this solution provokes an instantaneous change in the current waveform and therefore, in the applied torque. Others have evenly distributed this error along the time of the next Hall sector as suggest [8]. This last solution avoids the instantaneous change in the angle representation, but it continuously modifies the electrical speed in an electrical period.

In this paper, the electrical speed correction is only applied at the beginning of a new electrical period. That means at the moment in which the Hall sector is six or five at the anti-clockwise or clockwise direction, respectively. Then, an average correction is calculated once an electrical period as

$$
\begin{aligned}
& \omega_{e_{-} \operatorname{corr}(m)}=\frac{\left(\theta_{e(\operatorname{Hall}(z))}-\theta_{e(k)}\right)}{\Delta t_{(i-1)}+\Delta t_{(i-2)}+\Delta t_{(i-3)}} \cdot \frac{1}{2}, \\
& \forall \operatorname{Hall}(z)=5 \mid 6, \forall m>1, \forall i \geq 4 ;
\end{aligned}
$$

where $\omega_{\text {e_corr(m) }}$ is the electrical speed correction at each electrical period where the subscript $\mathrm{m}$ indicates the number of electrical periods. So, the electrical angle representation can be expressed as

$$
\begin{aligned}
\theta_{e(k)}=\theta_{e(k-1)}+\left(\omega_{e(i)}+\omega_{e_{\operatorname{corr}(m)}}\right) \cdot\left(t_{(k)}-\right. \\
\left.t_{(k-1)}\right), \\
\forall k \geq 1, \forall m>1, \forall i \geq 4 .
\end{aligned}
$$

With the usage of this method, the electrical angle is not saturated between the theoretical values during the transients, as it is proposed by other authors [8]. From now on, the proposed method in this paper is called as Method 1 and the explained method in [8] is called as Method 2.

\section{Simulation Results}

A Matlab-Simulink model is designed to validate the proposed method. It describes the behavior of an emotorbike, by gathering four systems: battery, power converter, electric motor and mechanical forces [9]. The power converter is modeled according to its average model in the abc reference, meanwhile the PMSM is modeled in the dq reference.

The control algorithm of this motor is based on the Maximum Torque per Ampere (MTPA) strategy because it is considered that the motor always works in the Low 


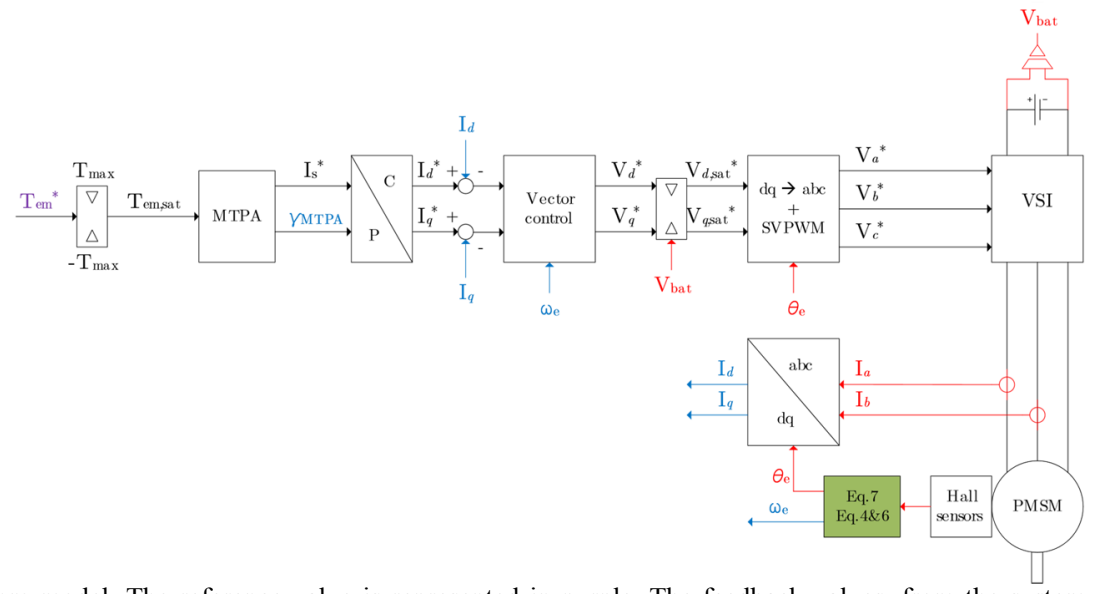

Fig. 5. Control algorithm model. The reference value is represented in purple. The feedback values, from the system, are shown in red. The intermediate calculations are depicted in blue. The speed calculation and the angle representation are indicated in the green block.

Back Electromotive Force (LBEF) zone and no FluxWeakening (FW) technique is required. A direct-drive IPMSM, which runs as the rear wheel of an e-motorbike, is used, and its characteristic parameters are listed in TABLE III.

TABLE III IPMSM CHARACTERISTIC PARAMETERS

\begin{tabular}{|l|r|r|}
\hline \multicolumn{1}{|c|}{ Symbol } & \multicolumn{1}{c|}{ Value } & \multicolumn{1}{c|}{ Unit } \\
\hline $\mathrm{R}_{\mathrm{s}}$ & 17 & $\mathrm{~m} \Omega$ \\
\hline $\mathrm{L}_{\mathrm{d}}$ & 70 & $\mu \mathrm{H}$ \\
\hline $\mathrm{L}_{\mathrm{q}}$ & 79 & $\mu \mathrm{H}$ \\
\hline$\lambda_{\mathrm{m}}$ & 0.023 & $(\mathrm{~V} \cdot \mathrm{s}) / \mathrm{rad}$ \\
\hline $\mathrm{V}_{\mathrm{bat}}$ & 48 & $\mathrm{~V}$ \\
\hline $\mathrm{I}_{\mathrm{s}, \max }$ & 467 & $\mathrm{~A}$ \\
\hline $\mathrm{pp}$ & 20 & \\
\hline
\end{tabular}

Fig. 5 shows the basis of the designed control. As can be seen, the electrical angle and speed affect:

1. Current conversion from abc to dq system-reference.

2. Reference voltages calculated by the PI controllers are converted from the dq to the abc system-reference.

3. Feed-forward PID current controllers, by adding the cross-terms which depend on the electrical speed.

Besides, the Back Electromotive Force is considered perfectly sinusoidal due to a sinusoidal winding distribution, so the current harmonic content can only appear due to an incorrect angle representation.

Fig. 6 shows the measurement of the induced voltages and the Hall sensor edges for each phase. In the experimental test, this should be done before the control is applied. The electric motor is mechanically powered up at $600 \mathrm{rpm}$. In this case, the phase-shift between the phaseneutral induced voltage and the Hall sensor signal for each phase is $+15^{\circ} \mathrm{E},-5^{\circ} \mathrm{E}$ and $+10^{\circ} \mathrm{E}$, respectively. Fig. 7 compares both the ideal and real electrical angle representation from the Method 1 and Method 2, respectively. Comparing the two pictures, it can be seen that the Method 2 generates an electrical angle with some harmonic content.

The load test is performed by applying the nominal torque, and considering the rated power and the rated mechanical speed of the motor of $2.5 \mathrm{~kW}$ and $510 \mathrm{rpm}$, respectively.
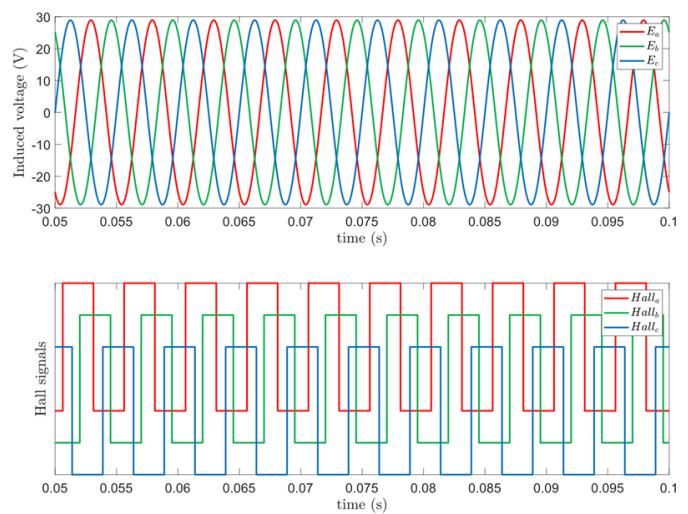

Fig. 6. Simulation results of the induced voltages and Hall sensor edges when the electric motor spin at $600 \mathrm{rpm}$ (mechanical speed) in generator mode.
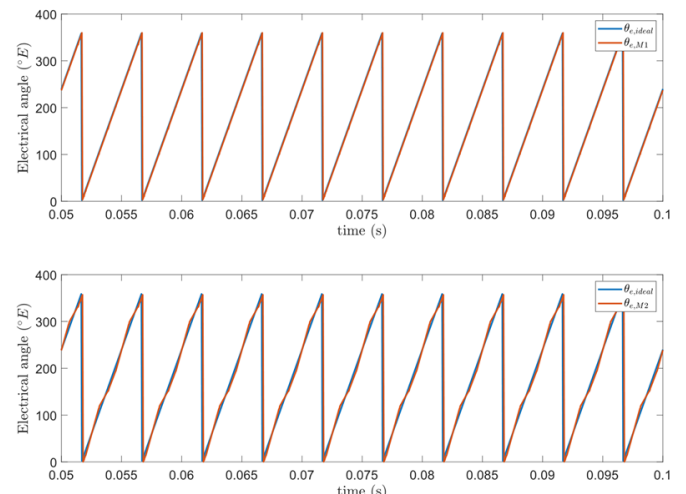

Fig. 7. Simulation results of electrical angle representation. It compares the ideal electric angle (blue line) with the real representation by using the Method 1 (orange line, top figure) and the Method 2 (orange line, bottom figure).

Both methods have been compared throughout the current total harmonic content and the root-mean-square error (RMSE), both of the electrical angle and the estimated electrical speed. TABLE IV illustrates the differences between the two methods. Both methods get zero error in the estimated electrical speed thanks to calculating it from the edges of one Hall sensor edges 
phase. However, the correction of the phase-shift in the electrical angle representation lets Method 1 to reduce the error of the electrical angle representation and so, to minimize the current THD content.

TABLE IV

ELECTRICAL ANGLE AND SPEED ESTIMATED ERROR

\begin{tabular}{|l|c|c|c|}
\cline { 2 - 4 } \multicolumn{1}{c|}{} & $\begin{array}{c}\text { RMSE } \\
\omega_{\mathrm{e}}(\%)\end{array}$ & $\begin{array}{c}\text { RMSE } \\
\theta_{\mathrm{e}}(\%)\end{array}$ & $\begin{array}{c}\text { THD } \\
\mathrm{I}_{\mathrm{x}}(\%)\end{array}$ \\
\hline Method 1 & 0 & 0.2 & 1.0 \\
\hline Method 2 & 0 & 2.2 & 17.0 \\
\hline
\end{tabular}

Top picture of Fig. 8 shows the difference between the estimated mechanical speed and its real value. Besides, the bottom picture of Fig. 8 shows the difference between the electrical angle representation and its real value. This test demonstrates that Method 1 commits the minimum error at steady-state.

Fig. 9 analyzes the difference between the current waveforms when the electrical angle is obtained as of Method 1 and Method 2. The misplacement of the Hall sensors is not well-corrected through the Method 2, whereas the Method 1 gets a sinusoidal waveform.
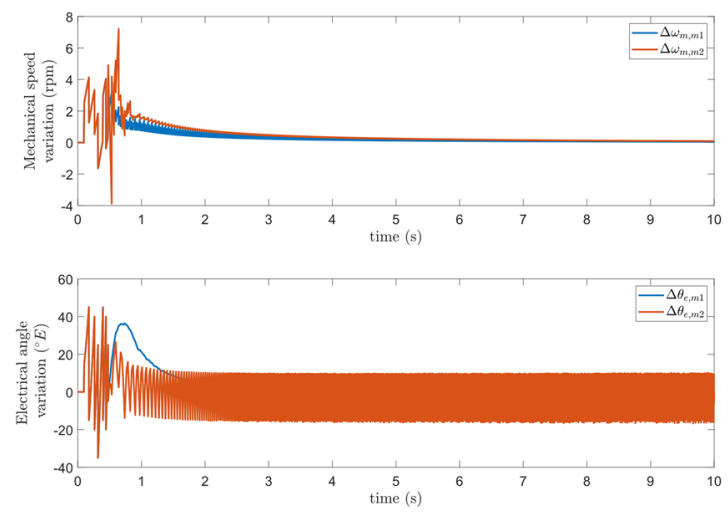

Fig. 8. Top figure: Estimated mechanical speed error calculated with Method 1 (blue line) and Method 2 (orange line). Bottom figure: Electrical angle error with Method 1 (blue line) and Method 2 (orange line).

\section{CONCLUSION}

This paper proposes an enhanced algorithm to represent the electrical angle from misplaced Hall-effect switch sensors to eliminate current and torque ripple. The algorithm is based on a First-Order Taylor-Series approximation which estimates the speed from two consecutive edges of the same phase and considers the phase-shift angle. In this paper, an IPMSM with a phaseshift of $+15^{\circ} \mathrm{E},-5^{\circ} \mathrm{E}$ and $+10^{\circ} \mathrm{E}$ for phase A, B and C Hall sensors have been considered. The proposed method achieves an electrical angle root-mean-square error below $0.5 \%$, and a current total harmonic distortion below $2 \%$.

\section{REFERENCES}

T. Finken, M. Felden, and K. Hameyer, "Comparison and design of different electrical machine types regarding their applicability in hybrid electrical vehicles," Proc. 2008 Int.
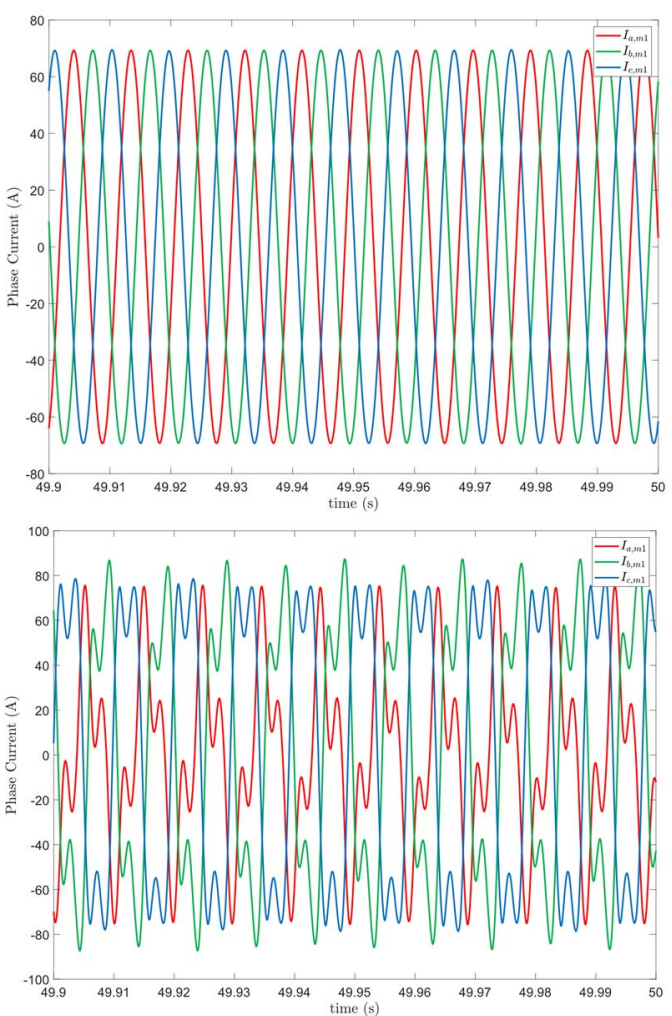

Fig. 9. Current waveforms of phase A, B and C at steady-state when the electrical angle is estimated using the Method 1 (top) and Method 2 (bottom)

[2] R. Bojoi, M. Pastorelli, J. Bottomley, P. Giangrande, and C. Gerada, "Sensorless control of PM motor drives - A technology status review," Proc. - 2013 IEEE Work. Electr. Mach. Des. Control Diagnosis, WEMDCD 2013, pp. 168182, 2013.

[3] F. Giulii Capponi, G. De Donato, L. Del Ferraro, O. Honorati, M. C. Harke, and R. D. Lorenz, "AC brushless drive with low-resolution hall-effect sensors for surface-mounted PM machines," IEEE Trans. Ind. Appl., vol. 42, no. 2, pp. 526535, 2006.

[4] L. Yu, Y. Zhang, and W. Huang, “Accurate and efficient torque control of an interior permanent magnet synchronous motor in electric vehicles based on Hall-effect sensors," Energies, vol. 10, no. 3, pp. 1-16, 2017.

[5] S. Kim, C. Choi, K. Lee, and W. Lee, "An Improved Rotor Position Estimation With Vector-Tracking Observer in PMSM Drives With Low-Resolution Hall-Effect Sensors,' IEEE Trans. Ind. Electron., vol. 58, no. 9, pp. 4078-4086, 2011.

[6] J. A. Cortajarena, S. García, J. Cortajarena, O. Barambones, and P. Alkorta, "Influence of the rotor angle precision in control of interior permanent magnet synchronous machine drives and improvement method using sensorless estimator with Hall sensors," IET Power Electron., vol. 12, no. 3, pp. 383-391, 2019.

[7] X. Zhang and W. Zhang, "An Improved Rotor Position Estimation in PMSM with Low-Resolution Hall-Effect Sensors," in 2014 17th International Conference on Electrical Machines and Systems, ICEMS, 2014, pp. 2722-2727.

[8] L. Kreindler, I. Iacob, G. Casaru, A. Sarca, R. Olteanu, and D. Matianu, "PMSM drive using digital hall position sensors for light EV applications," in 2015 9th International Symposium on Advanced Topics in Electrical Engineering, ATEE 2015, 2015, pp. 199-204.

[9] D. Montesinos-Miracle and T. Ruiz-Bassols, "Regenerative braking in electric scooters," in 2017 19th European Conference on Power Electronics and Applications, EPE 2017 ECCE Europe, 2017, vol. 2017-Janua, pp. 1-8. 